\title{
A Simplified Identification Method of Dynamic Stiffness for the Heavy-Load and Low- Speed Journal Bearings
}

\author{
Junning LI, Ka HAN, Wei CHEN
}

\begin{abstract}
Journal bearing is an essential part of the propulsion shafting system, whose performance directly affects the safety and performance of the shaft and even the entire ship. It is an important, but also a difficult task to obtain the accurate dynamic stiffness values. In order to determine the dynamic characteristics of the journal bearing with specific structural characteristics under specific working conditions, an inverted test platform with multiple sensors for the dynamic stiffness of the heavy-load, low-speed journal bearings (HLLSJBs) was developed, and a novel dynamic test system calibration method was proposed to obtain accurate baseline data, and then the effective characteristic frequency response was gotten based on a refined fast Fourier transform method (RFFT). The present study proposed a simplified identification method to analyze the constitutive relationships between the oil film dynamic parameters and the dynamic stiffness under different rotation and loading conditions in the journal bearing, and then the accurate values of the oil film dynamic stiffness of HLLSJBs were obtained. Furthermore, the values of the structural dynamic stiffness of HLLSJBs were also obtained using a hammer impact method. A comparison with the classic theoretical analysis results reveals that the method proposed is more accurate and effective.
\end{abstract}

Keywords: journal bearing; multiple sensors; oil film dynamic stiffness; propulsion shafting system; structural dynamic stiffness

\section{INTRODUCTION}

Bearings are important components commonly used in the rotating machinery parts of trains, ships, and automobile manufacturing, among others [1]. The vibration and stability of rotating machinery mainly determine its journal bearings, and the most important indicators for failure prediction in journal bearings are the analysis of the dynamic behaviour of the bearing $[2,3]$. Detailed numerical values of the bearing dynamic characteristics are necessary for proper design and operation of rotating machinery $[4,5]$. Improper design of such bearings can lead to a variety of faults and even failure of the whole system [6]. Thus, it is the basis for making accurate predictions of the dynamic characteristics of rotor-bearing systems that properly determine the dynamic characteristic of a journal bearing. Propulsion shafting system plays a pivotal role in the normal and stable operation of a large ship [7]. The main journal bearing is a key part of the propulsion shafting system, whose performance directly affects the safety and performance of the shaft and even the entire ship [8]. Tiwari [9] introduced a method for identification of bearing dynamic parameters from impulse responses of rotor-bearing systems. Żywica [10] presented the experimental investigation so as to identify bearing dynamic coefficients of two hydrodynamic bearings from impulse responses. Schwartz [11] investigated the effect of systematic errors on the accuracy of experimental measurements of fluid-film bearing dynamic coefficients. Li [12] proposed a method for the numerical identification of bearing support force coefficients in flexible rotor-bearing systems based on the 3D transient flow calculation. Song [13] presented an experimental method for identifying the dynamic coefficients of the oil-film in a journal bearing, including the oil-film stiffness and damping coefficients based on vibration theory and the least squares method; as a result, particular models can be established, and the associated experimental research can be undertaken. Yuan [14] introduced a method to determine the oil film stiffness and damping coefficients by use of the multi-frequency technique; the method also selected electro-mechanical coupling to produce an unstable system on the same testing rig to evaluate the accuracy of the testing result. Lin [15] investigated the various aspects that influence the accuracy of measurement, and then several preliminary conclusions were made from the investigation. The gain factors and phase factors of the frequency response functions of a test system are different for different channels, so the use of a dynamic calibration procedure is highly necessary.

Because the dynamic stiffness of HLLSJBs is essentially nonlinear [16], the acquisition of the accurate dynamic stiffness values has always been a difficult primary objective, which involves the use of multi-sensor information such as displacement, speed, acceleration and force. Hence, the present study proposes a simplified method for identifying the dynamic stiffness of the journal bearings, so as to determine the dynamic performance of the journal bearings of the propulsion shafting systems in ships with specific structural characteristics under specific working conditions.

\section{MATERIALS AND METHODS \\ 2.1 Structure of the Test Platform}

In order to obtain the dynamic coefficients of journal bearings with specific structural characteristics under specific working conditions, a test platform with multiple sensors for determining the dynamic stiffness of HLLSJBs was developed. Test platform has an inverted structure and consists mainly of two parts, a test bench and a test system. Test bench consists mainly of a driving device, loading and excitation devices, a lubricating device, the main shaft and test bearing components. Test system consists mainly of displacement and force sensors and a data acquisition and processing system. Fig. 1 shows the structure of the test bench.

1) Driving device

The driving device consists mainly of a motor, a gearbox that can increase the system's speed and a coupling. A variable-frequency, variable-speed motor with a continuously variable rotational speed is used. The rotational speed of the motor is controlled using a frequency modulator to change from low rotational speeds 
to the rotational speeds required for the test. A flange coupling is used. A gap is preserved between the test main shaft and the coupling in the axial direction of the shaft. The two flanges are tied using nylon ropes so that the torque is transmitted to the test main shaft, thereby isolating the vibration of the speed-increasing gearbox as much as possible.

2) Loading and excitation devices

The stiffness and damping coefficients of a bearing are related to the load that the bearing endures. Therefore, it is necessary to apply two types of load to the test bearing: a static load (to simulate the external load that the actual bearing endures) and an exciting force (to simulate the disturbing force to which the bearing is subjected).

The static load is applied to the bearing by pumping compressed air using the corrugated pipe. The corrugated pipe is installed below the test bearing to apply a vertical static load onto the test bearing.

The exciting force on the bearing is provided by exciters. Two electromagnetic exciters are installed on the test platform. The exciters are placed in such a way that they are symmetric about the axial direction of the test bearing. The angle between the axis of each exciter and the horizontal plane is $45^{\circ}$. This way, two exciting forces that are vertical to each other and pass the geometric centre of the bearing can be applied to minimize the interference between the forces and improve the precision and accuracy of signal acquisition.

3) Lubricating device

The function of the lubricating device is to provide a lubricant for the test bearing. The lubricating device consists mainly of an oil supply station, an oil pump, a filter, a valve, a pressure gauge, a heat exchanger, a pipeline, a return oil tank, and a return oil pump. The oil supply station is placed at a certain distance from the test platform and connected to the test platform via a preinstalled pipeline.

4) Main shaft and test bearing components

The test shaft is made of 45 steels. Through various processing treatments, it is ensured that the shaft has high concentricity and surface roughness. The main shaft is supported by two high-precision radial ball bearings. In addition, the runout of the main shaft when it rotates is minimized.

The test bearing is installed on the test bearing sleeves and can float on the rotating main shaft. The floating test bearing is bidirectionally axially positioned using eight iron chains.

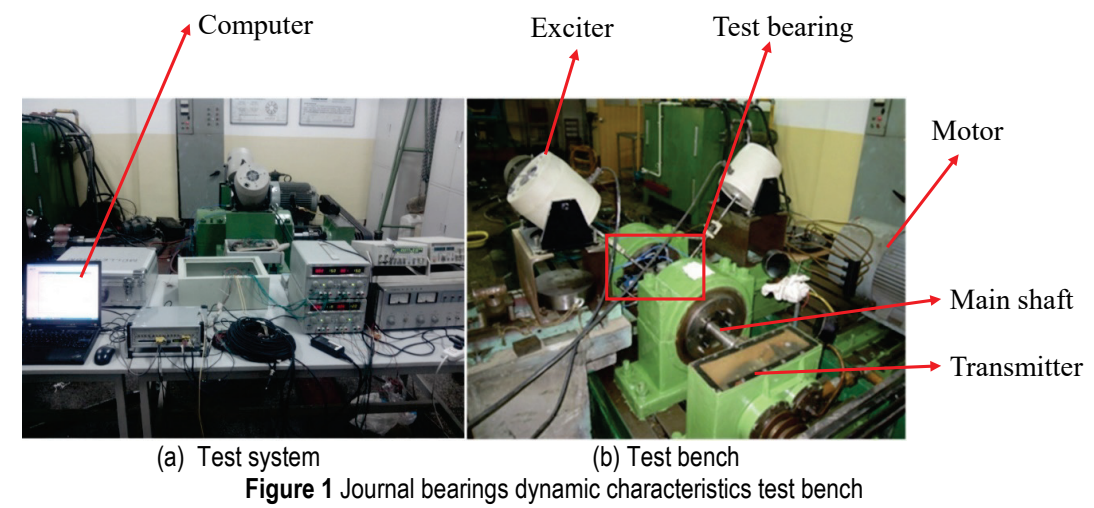

\subsection{Test System and its Working Principle for the Oil Film Dynamic Stiffness of HLLSJBs}

1) Test system with multiple sensors

The test system for the oil films dynamic stiffness consists mainly of displacement, force and temperature sensors and a data acquisition and processing device and is used to obtain load and displacement signals under specific working conditions. The sensor at the front end of the test system converts the tested physical quantity to an electrical signal, which, after being conditioned and amplified by a transmitter, is converted into an analog signal compatible with the acquisition instrument. Supported by the data acquisition and processing system at the back end, the test system converts multi-channel analog signals into digital signals and stores these signals to facilitate the analysis and processing of the test results.

The relative displacement signal of the test bearing is measured in two directions, vertical and horizontal; the absolute displacement signal of the test bearing is also measured in two directions, vertical and horizontal (Fig. 2).

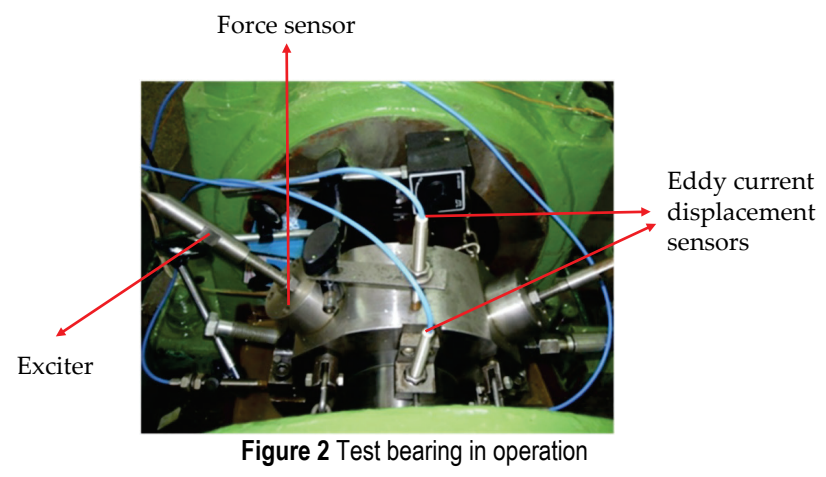

Test is conducted on an inverted test platform. Fig. 3 shows the mechanical model of the test bearing system. Here, it is assumed that the bearing is a point mass and that the oil film is a linear spring and a viscous damper. In addition, it is considered that the vectors of the applied forces pass through the geometric centre of the bearing. Then, the test bearing system can be viewed as a linear system consisting of a point mass shaft, a spring and a damper. The dynamic characteristic of the test bearing can be obtained using the complex excitation method.

The relative displacement signal of the test bearing is measured in two directions, vertical and horizontal; the 
absolute displacement signal of the test bearing is also measured in two directions, vertical and horizontal (Fig. 3 and 4).

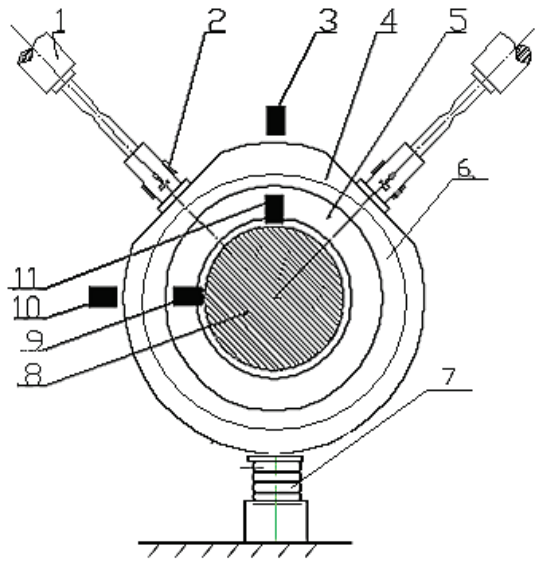

Figure 3 Layout of the measuring points of the displacement signal of the test bearing (1 - exciter; 2 - force sensor; 3, 9, 10 and 11 - eddy current displacement sensors; 4 and 6 - test bearing sleeves; 5 - test bearing; 6 supporting ball bearing; 7 - corrugated pipe; 8 - test main shaft)

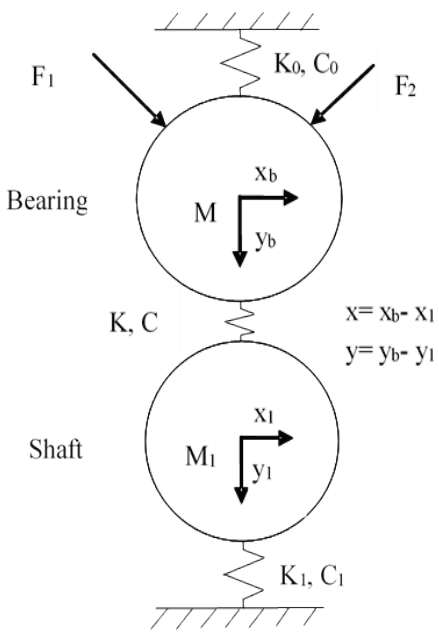

Figure 4 Mechanical model of the test bearing system $\left(M\right.$ - mass; $x_{b}$ and $y_{b}$ displacements of the test bearing; $x_{1}$ and $y_{1}$ - displacements of the rotating main shaft; $K$ - oil film stiffness; $C$ - oil film damping coefficient; subscript 1 - rotating main shaft; subscript b-test bearing; subscript 0 - the base)

2) Dynamic calibration of the test system

A new dynamic calibration method is used. The core of this method lies in the fact that each channel of the test system is dynamically calibrated directly on the test platform with the floating bearing which is used as the mass block for calibration. When no force is applied to the corrugated pipe, the floating test bearing falls on the main shaft. An air compressor is used to apply force to the corrugated pipe to allow the floating test bearing to slowly float. The position of the floating test bearing is then adjusted to allow the shaft to separate from the bearing and the bearing to reside at the central location as much as possible. The directions of the two ends of the floating bearing on the test platform are fixed by guiding devices. The mass of the floating bearing is known. This method can ensure that the calibration conditions may be consistent with the test conditions, which is beneficial to the improvement of the accuracy and precision of the test results.

3) Simplified method for identifying dynamic characteristics

Using the inverted test platform shown in Fig. 1, exciting forces are applied to the floating test bearing, and the response of the floating test bearing is measured. Then, the oil film stiffness and damping coefficients can be determined using motion Eq. (1):

$$
M \ddot{\boldsymbol{X}}_{0}+C \dot{\boldsymbol{X}}+K \boldsymbol{X}=F(t)
$$

where $M$ - mass of the floating bearing,

$M=\left[\begin{array}{cc}M & 0 \\ 0 & M\end{array}\right]$

$C$ - damping coefficient of the oil film,

$C=\left[\begin{array}{ll}C_{x x} & C_{x y} \\ C_{y x} & C_{y y}\end{array}\right]$

$K$ - stiffness coefficient of the oil film,

$K=\left[\begin{array}{ll}K_{x x} & K_{x y} \\ K_{y x} & K_{y y}\end{array}\right]$

$\ddot{X}_{0}$ - acceleration vector of the floating bearing,

$\ddot{\boldsymbol{X}}_{0}=\left[\begin{array}{l}\ddot{x}_{0} \\ \ddot{y}_{0}\end{array}\right]$

$\boldsymbol{X}$ and $\dot{\boldsymbol{X}}$ - displacement vector and speed vector of the relative journal of the floating bearing, respectively,

$\boldsymbol{X}=\left[\begin{array}{l}x \\ y\end{array}\right], \dot{\boldsymbol{X}}=\left[\begin{array}{l}\dot{x} \\ \dot{y}\end{array}\right]$

$F(t)$ - component of the exciting force.

As shown in Fig. $4, F_{1}$ has an excitation frequency of $\omega_{1}$, and $F_{2}$ has an excitation frequency of $\omega_{2}$. Based on Eq. (1) and the coordinate system, a motion equation is established:

$$
\left\{\begin{array}{l}
K_{x x}\left(x_{1}-x_{2}\right)+C_{x x}\left(\dot{x}_{1}-\dot{x}_{2}\right)+K_{x y}\left(-y_{1}-y_{2}\right)+C_{x y}\left(-\dot{y}_{1}-\dot{y}_{2}\right)+M\left(\ddot{x}_{01}-\ddot{x}_{02}\right)=F_{1 x}(t)-F_{2 x}(t) \\
K_{y y}\left(-y_{1}-y_{2}\right)+C_{y y}\left(-\dot{y}_{1}-\dot{y}_{2}\right)+K_{y x}\left(x_{1}-x_{2}\right)+C_{y x}\left(\dot{x}_{1}-\dot{x}_{2}\right)+M\left(-\ddot{y}_{01}-\ddot{y}_{02}\right)=-F_{1 y}(t)-F_{2 y}(t)
\end{array}\right.
$$

Eq. (7) is subjected to a Fourier transform. Because the corresponding values of the two frequencies in the frequency domain are independent from each other, four equations can be obtained: 
$\left\{\begin{array}{l}j \omega_{1} C_{x x} X_{1}-j \omega_{1} C_{x y} Y_{1}+K_{x x} X_{1}-K_{x y} Y_{1}=F_{1 x}\left(\omega_{1}\right)+\omega_{1}^{2} M X_{01} \\ -j \omega_{1} C_{y x} X_{1}+j \omega_{1} C_{y y} Y_{1}-K_{y x} X_{1}+K_{y y} Y_{1}=F_{1 y}\left(\omega_{1}\right)+\omega_{1}^{2} M Y_{01} \\ j \omega_{2} C_{x x} X_{2}+j \omega_{2} C_{x y} Y_{2}+K_{x x} X_{2}+K_{x y} Y_{2}=F_{2 x}\left(\omega_{2}\right)+\omega_{2}^{2} M X_{02} \\ j \omega_{2} C_{y x} X_{2}+j \omega_{2} C_{y y} Y_{2}+K_{y x} X_{2}+K_{y y} Y_{2}=F_{2 y}\left(\omega_{2}\right)+\omega_{2}^{2} M Y_{02}\end{array}\right.$

Eq. (8) is further treated:

$$
\left\{\begin{array}{l}
j \omega_{1} C_{x x} \frac{X_{1}}{F_{1 x}\left(\omega_{1}\right)}-j \omega_{1} C_{x y} \frac{Y_{1}}{F_{1 x}\left(\omega_{1}\right)}+K_{x x} \frac{X_{1}}{F_{1 x}\left(\omega_{1}\right)}-K_{x y} \frac{Y_{1}}{F_{1 x}\left(\omega_{1}\right)}=1+\omega_{1}^{2} M \frac{X_{01}}{F_{1 x}\left(\omega_{1}\right)} \\
-j \omega_{1} C_{y x} \frac{X_{1}}{F_{1 y}\left(\omega_{1}\right)}+j \omega_{1} C_{y y} \frac{Y_{1}}{F_{1 y}\left(\omega_{1}\right)}-K_{y x} \frac{X_{1}}{F_{1 y}\left(\omega_{1}\right)}+K_{y y} \frac{Y_{1}}{F_{1 y}\left(\omega_{1}\right)}=1+\omega_{1}^{2} M \frac{Y_{01}}{F_{1 y}\left(\omega_{1}\right)} \\
j \omega_{2} C_{x x} \frac{X_{2}}{F_{2 x}\left(\omega_{2}\right)}+j \omega_{2} C_{x y} \frac{Y_{2}}{F_{2 x}\left(\omega_{2}\right)}+K_{x x} \frac{X_{2}}{F_{2 x}\left(\omega_{2}\right)}+K_{x y} \frac{Y_{2}}{F_{2 x}\left(\omega_{2}\right)}=1+\omega_{2}^{2} M \frac{X_{02}}{F_{2 x}\left(\omega_{2}\right)} \\
j \omega_{2} C_{y x} \frac{X_{2}}{F_{2 y}\left(\omega_{2}\right)}+j \omega_{2} C_{y y} \frac{Y_{2}}{F_{2 y}\left(\omega_{2}\right)}+K_{y x} \frac{X_{2}}{F_{2 y}\left(\omega_{2}\right)}+K_{y y} \frac{Y_{2}}{F_{2 y}\left(\omega_{2}\right)}=1+\omega_{2}^{2} M \frac{Y_{02}}{F_{2 y}\left(\omega_{2}\right)}
\end{array}\right.
$$

For the test results, the force sensors are used as the reference, and their results are set to positive. Thus, the surface of each eddy current sensor oriented away from the test is positive. The signs of the sensor test results under frequencies $\omega_{1}$ and $\omega_{2}$ are shown in Tab. 1.

Table 1 Signs of the sensor test results under different frequencies

\begin{tabular}{|l|c|c|}
\hline \multicolumn{1}{|c|}{ Sensor location } & $\omega_{1}$ & $\omega_{2}$ \\
\hline Relative $y$-axis & - & - \\
\hline Relative $x$-axis & - & + \\
\hline Absolute $y$-axis & + & + \\
\hline Absolute $x$-axis & - & + \\
\hline
\end{tabular}

By substituting the signs listed in Tab. 1 into Eq. (9),

$$
\left\{\begin{array}{l}
-j \omega_{1} C_{x x} \frac{X_{1}}{F_{1 x}\left(\omega_{1}\right)}+j \omega_{1} C_{x y} \frac{Y_{1}}{F_{1 x}\left(\omega_{1}\right)}-K_{x x} \frac{X_{1}}{F_{1 x}\left(\omega_{1}\right)}+K_{x y} \frac{Y_{1}}{F_{1 x}\left(\omega_{1}\right)}=1-\omega_{1}^{2} M \frac{X_{01}}{F_{1 x}\left(\omega_{1}\right)} \\
j \omega_{1} C_{y x} \frac{X_{1}}{F_{1 y}\left(\omega_{1}\right)}-j \omega_{1} C_{y y} \frac{Y_{1}}{F_{1 y}\left(\omega_{1}\right)}+K_{y x} \frac{X_{1}}{F_{1 y}\left(\omega_{1}\right)}-K_{y y} \frac{Y_{1}}{F_{1 y}\left(\omega_{1}\right)}=1+\omega_{1}^{2} M \frac{Y_{01}}{F_{1 y}\left(\omega_{1}\right)} \\
j \omega_{2} C_{x x} \frac{X_{2}}{F_{2 x}\left(\omega_{2}\right)}-j \omega_{2} C_{x y} \frac{Y_{2}}{F_{2 x}\left(\omega_{2}\right)}+K_{x x} \frac{X_{2}}{F_{2 x}\left(\omega_{2}\right)}-K_{x y} \frac{Y_{2}}{F_{2 x}\left(\omega_{2}\right)}=1+\omega_{2}^{2} M \frac{X_{02}}{F_{2 x}\left(\omega_{2}\right)} \\
j \omega_{2} C_{y x} \frac{X_{2}}{F_{2 y}\left(\omega_{2}\right)}-j \omega_{2} C_{y y} \frac{Y_{2}}{F_{2 y}\left(\omega_{2}\right)}+K_{y x} \frac{X_{2}}{F_{2 y}\left(\omega_{2}\right)}-K_{y y} \frac{Y_{2}}{F_{2 y}\left(\omega_{2}\right)}=1+\omega_{2}^{2} M \frac{Y_{02}}{F_{2 y}\left(\omega_{2}\right)}
\end{array}\right.
$$

Based on the frequency response function of the test system obtained through the dynamic calibration of the test system $H_{\text {System }}=\frac{X}{F}=\frac{\frac{X_{\text {Measured }}}{F_{\text {Measured }}}}{H_{\text {Calibrated }}}$, Eq. (10) can be adjusted to:

$$
\begin{aligned}
& \int-j \omega_{1} C_{x x} \frac{\frac{X_{1}}{F_{1 x}\left(\omega_{1}\right)}}{H_{12}}+j \omega_{1} C_{x y} \frac{\frac{Y_{1}}{F_{1 x}\left(\omega_{1}\right)}}{H_{13}}-K_{x x} \frac{\frac{X_{1}}{F_{1 x}\left(\omega_{1}\right)}}{H_{12}}+K_{x y} \frac{\frac{Y_{1}}{F_{1 x}\left(\omega_{1}\right)}}{H_{13}}=1-\omega_{1}^{2} M \frac{\frac{X_{01}}{F_{1 x}\left(\omega_{1}\right)}}{H_{11}} \\
& j \omega_{1} C_{y x} \frac{\frac{X_{1}}{F_{1 y}\left(\omega_{1}\right)}}{H_{12}}-j \omega_{1} C_{y y} \frac{\frac{Y_{1}}{F_{1 y}\left(\omega_{1}\right)}}{H_{13}}+K_{y x} \frac{\frac{X_{1}}{F_{1 y}\left(\omega_{1}\right)}}{H_{12}}-K_{y y} \frac{\frac{Y_{1}}{F_{1 y}\left(\omega_{1}\right)}}{H_{13}}=1+\omega_{1}^{2} M \frac{\frac{Y_{01}}{F_{1 y}\left(\omega_{1}\right)}}{H_{14}} \\
& \left\{j \omega_{2} C_{x x} \frac{\frac{X_{2}}{F_{2 x}\left(\omega_{2}\right)}}{H_{22}}-j \omega_{2} C_{x y} \frac{\frac{Y_{2}}{F_{2 x}\left(\omega_{2}\right)}}{H_{23}}+K_{x x} \frac{\frac{X_{2}}{F_{2 x}\left(\omega_{2}\right)}}{H_{22}}-K_{x y} \frac{\frac{Y_{2}}{F_{2 x}\left(\omega_{2}\right)}}{H_{23}}=1+\omega_{2}^{2} M \frac{\frac{X_{02}}{F_{2 x}\left(\omega_{2}\right)}}{H_{21}}\right. \\
& j \omega_{2} C_{y x} \frac{\frac{X_{2}}{F_{2 y}\left(\omega_{2}\right)}}{H_{22}}-j \omega_{2} C_{y y} \frac{\frac{Y_{2}}{F_{2 y}\left(\omega_{2}\right)}}{H_{23}}+K_{y x} \frac{\frac{X_{2}}{F_{2 y}\left(\omega_{2}\right)}}{H_{22}}-K_{y y} \frac{\frac{Y_{2}}{F_{2 y}\left(\omega_{2}\right)}}{H_{23}}=1+\omega_{2}^{2} M \frac{\frac{Y_{02}}{F_{2 y}\left(\omega_{2}\right)}}{H_{24}}
\end{aligned}
$$

where $H_{11}, H_{12}, H_{13}$ and $H_{14}$ represent the frequency response functions of the test system under the exciting force with a frequency of $\omega_{1}$ obtained through calibration with respect to the absolute $x$-axis, the relative $x$-axis, the relative $y$-axis and the absolute $y$-axis, respectively; $H_{21}$, $\mathrm{H}_{22}, \mathrm{H}_{23}$ and $\mathrm{H}_{24}$ represent the frequency response functions of the test system under the exciting force with a frequency of $\omega_{2}$ obtained through calibration with respect to the absolute $x$-axis, the relative $x$-axis, the relative $y$-axis and the absolute $y$-axis, respectively.

By taking the real and imaginary parts of each equality in Eq. (11)

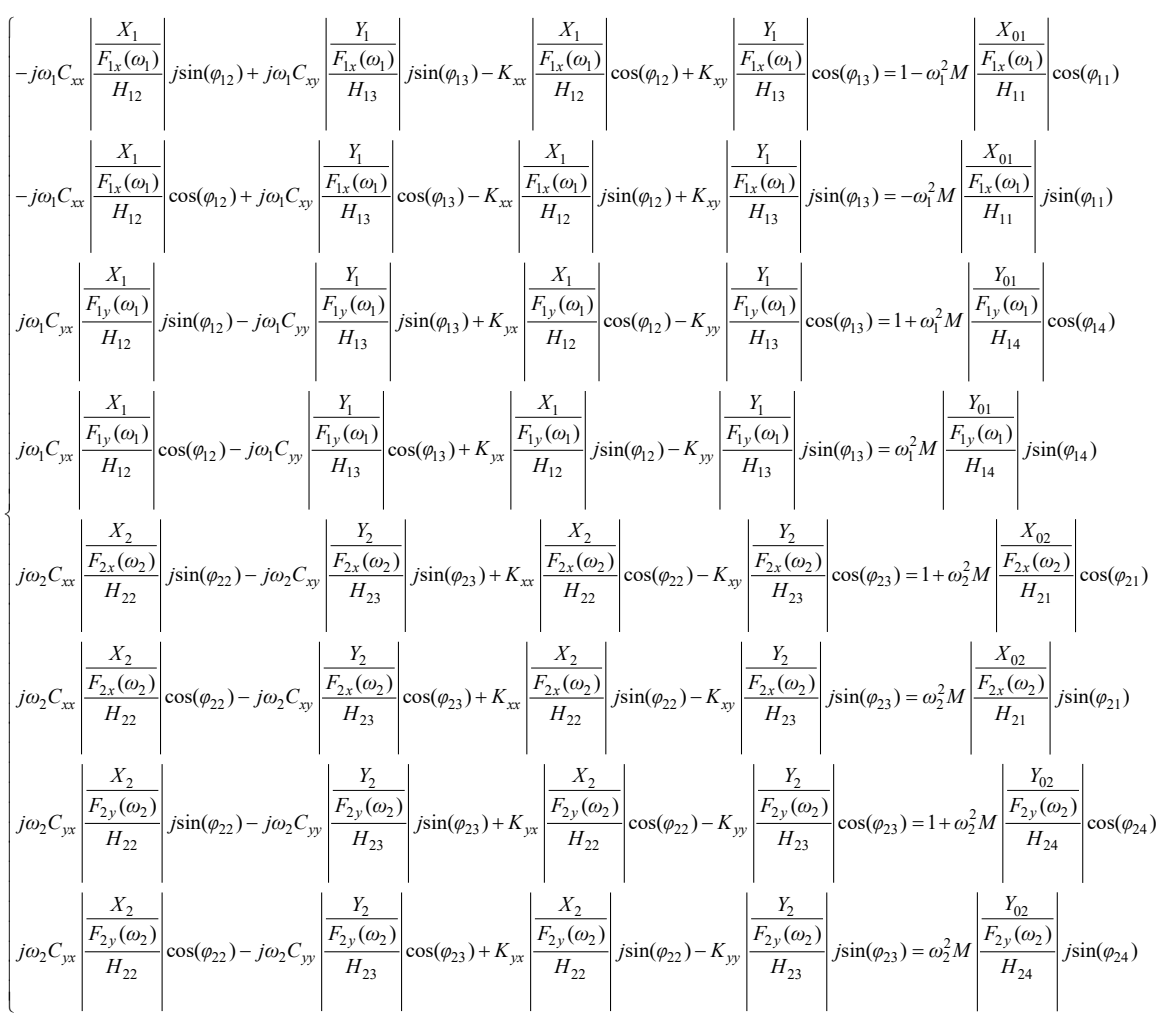


where $\varphi_{11}, \varphi_{12}, \varphi_{13}$ and $\varphi_{14}$ represent the phase angles of the frequency response functions of the system under the exciting force with a frequency of $\omega_{1}$ determined by the test system and treated against the calibration results with respect to the absolute $x$-axis, the relative $x$-axis, the relative $y$-axis and the absolute y-axis, respectively; $\varphi_{21}$, $\varphi_{22}, \varphi_{23}$ and $\varphi_{24}$ represent the phase angles of the frequency response functions of the test system under the exciting force with a frequency of $\omega_{2}$ determined by the test system and treated against the calibration results with respect to the absolute $x$-axis, the relative $x$-axis, the relative $y$-axis and the absolute $y$-axis, respectively. Phase angle of the frequency response function of the system is:

$\varphi=\varphi_{X \text { measured }}-\varphi_{F \text { measured }}-\varphi_{\text {calibrated }}$

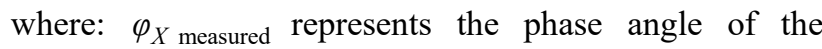
measured displacement response signal; $\varphi_{F \text { measured }}$ represents the phase angle of the measured force signal; and, $\varphi_{\text {calibrated }}$ represents the phase angle of the frequency response function of the test system obtained through calibration.

Eq. (12) is then written in the form of a matrix equality $(\boldsymbol{A} \boldsymbol{X}=\boldsymbol{B})$, which is simplified and adjusted to obtain an equation coefficient matrix. And then the parameter matrix to be identified is:

$$
\boldsymbol{X}=\left[\begin{array}{l}
K_{x x} \\
K_{x y} \\
C_{x x} \\
C_{x y} \\
K_{y x} \\
K_{y y} \\
C_{y x} \\
C_{y y}
\end{array}\right]
$$

Matrix $\boldsymbol{B}$ is give by Eq. (15).

By solving equation $\boldsymbol{A} \boldsymbol{X}=\boldsymbol{B}$, a total of eight parameters (stiffness and damping coefficients) of the oil film in the journal bearing on the test platform can be obtained.

The phase and amplitude data of the frequency response of different channels of the test system under each excitation required for the aforementioned program for identifying dynamic coefficients are obtained using a selfdeveloped interpolated Fourier transform algorithm and software. The software is programmed based on the GUIDE function of MATLAB and has an excellent manmachine interactive function. Owing to length limitations, the details of this software are not discussed in this article.

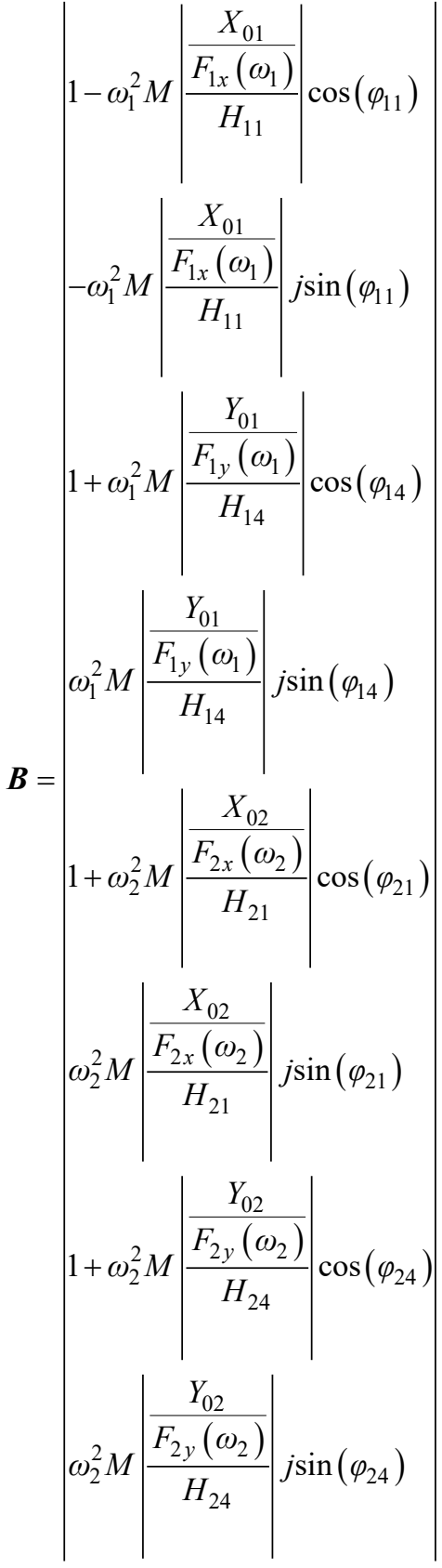

\subsection{Test System for the Structural Dynamic Stiffness of HLLSJBS}

Test system for the structural dynamic stiffness of HLLSJBs consists of a force hammer, a signalconditioning module, acceleration sensors, and a signal acquisition device and acquisition software.

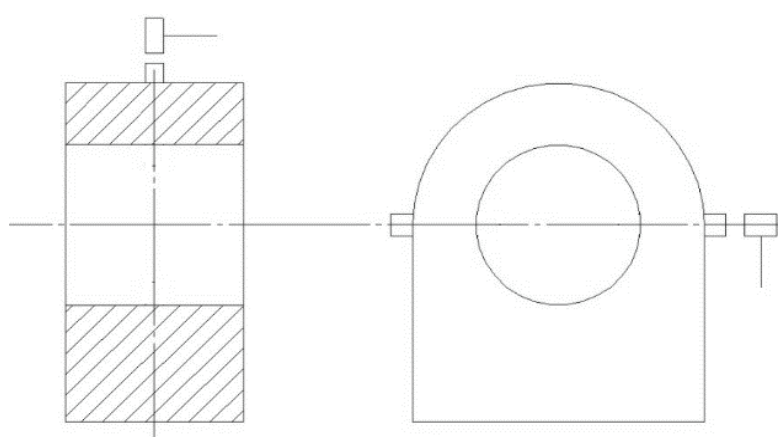

Figure 5 Structural dynamic stiffness test system 
Two acceleration sensors are placed along the external surface of the bearing base, and the arrangement of the sensors is shown in Fig. 5. The structural dynamic stiffness parameters of the structure are obtained by the hammer impact method.

\section{RESULTS AND DISCUSSION 3.1 Oil Film Dynamic Stiffness of HLLSJBs}

Refined testing is the key to the identification of the dynamic characteristics of the journal bearing. The sensor data (displacement and force signals) acquired under the specific working conditions are subjected to a RFFT to obtain the values of the amplitude and phase of the response of various displacement signals under the specific exciting force frequencies, together with the information of the amplitude and phase of the exciting forces, which are substituted into the dynamic stiffness identification program for data processing. The oil film dynamic stiffness and damping coefficients of the journal bearing can be obtained using the complex excitation method.

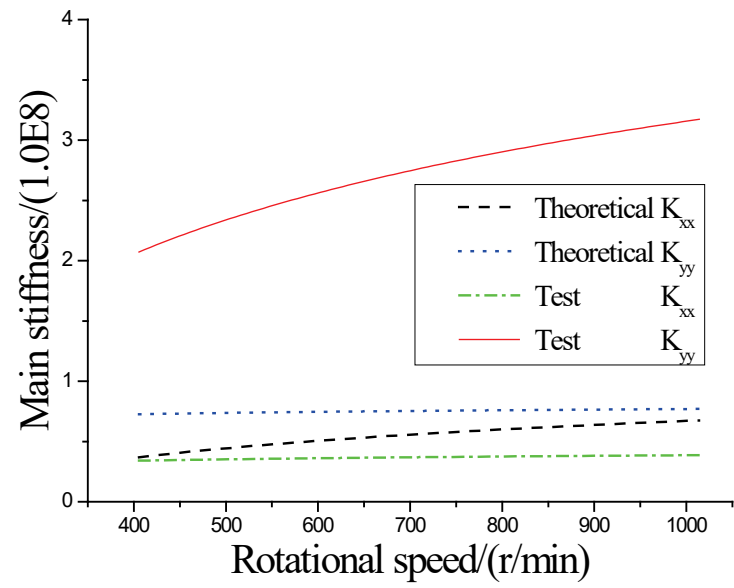

Figure 6 Main stiffness coefficient under different rotational speeds

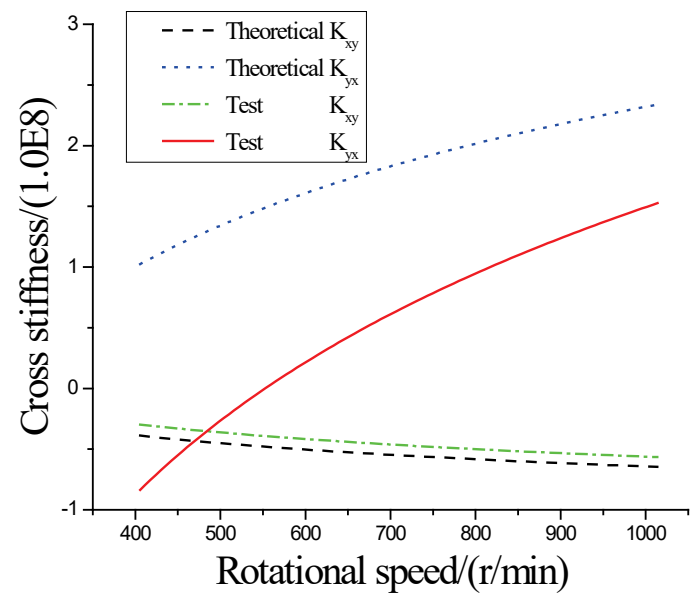

Figure 7 Cross stiffness coefficient under different rotational speeds

The test journal bearing length is $290 \mathrm{~mm}$, journal diameter is $370 \mathrm{~mm}$, the diametral clearance is $0.4 \mathrm{~mm}$, oil inlet temperature is $35^{\circ} \mathrm{C}$, and the oil inlet pressure is 0.2 MPa. Fig. 6-9 show the analysis results of the relationships between the rotational speed and the oil film dynamic stiffness and damping coefficients (load $=0.36 \mathrm{MPa}$ ). Fig. 10-13 show the analysis results of the relationships between the load and the oil film dynamic stiffness and damping coefficients (rotational speed $=811.2 \mathrm{r} / \mathrm{min}$ ). The test results under the specific working conditions and the classic theoretical calculation results are shown in Fig. 613. Under the specific working conditions, the oil film dynamic stiffness in the journal bearing has an order of magnitude of $10^{8} \mathrm{~N} / \mathrm{m}$. The results show that the values of the dynamic stiffness of the journal bearing obtained using the simplified identification method basically agree with the classic theoretical analysis results, thereby verifying the accuracy of the proposed method.

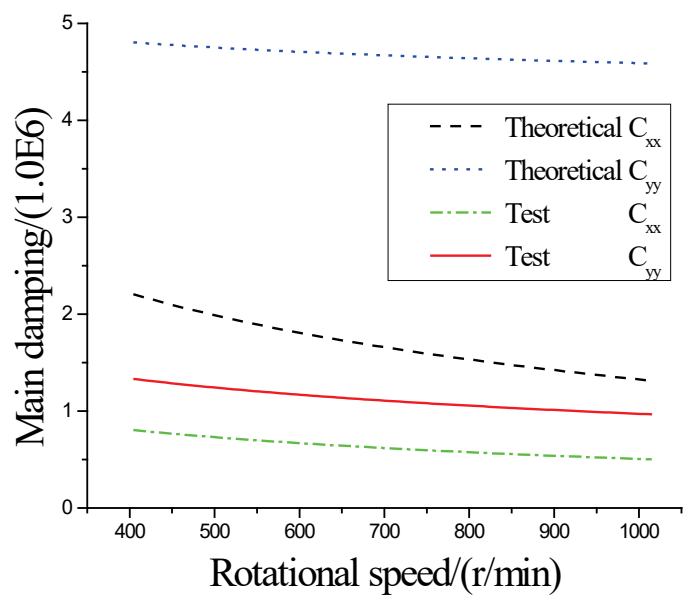

Figure 8 Main damping coefficient under different rotational speeds

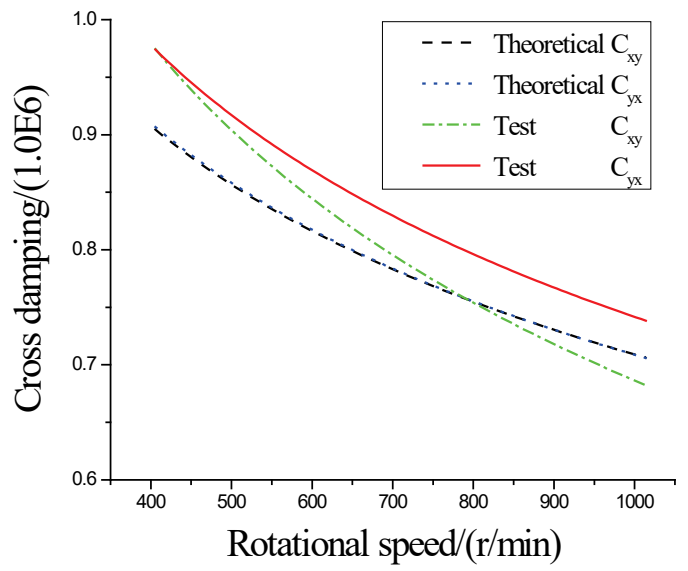

Figure 9 Cross damping coefficient under different rotational speeds

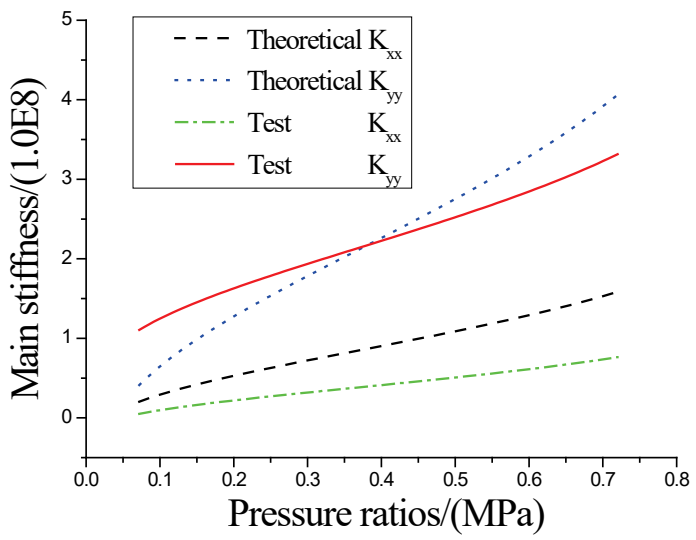

Figure 10 Main stiffness coefficient under different pressure ratios 


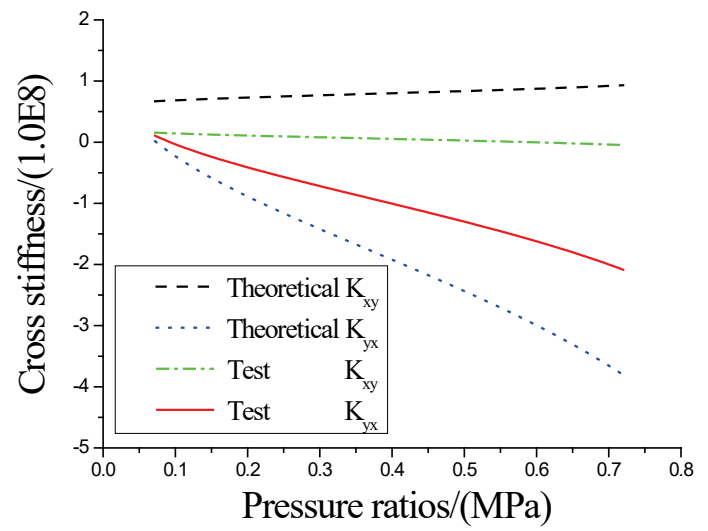

Figure 11 Cross stiffness coefficient under different pressure ratios

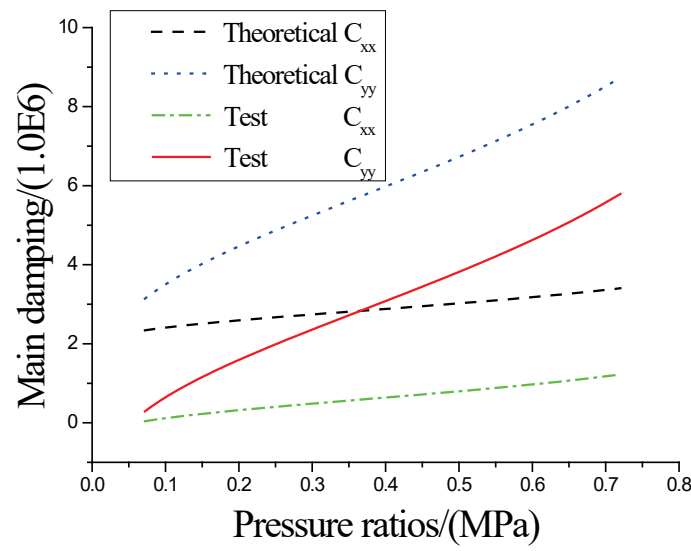

Figure 12 Main damping coefficient under different pressure ratios

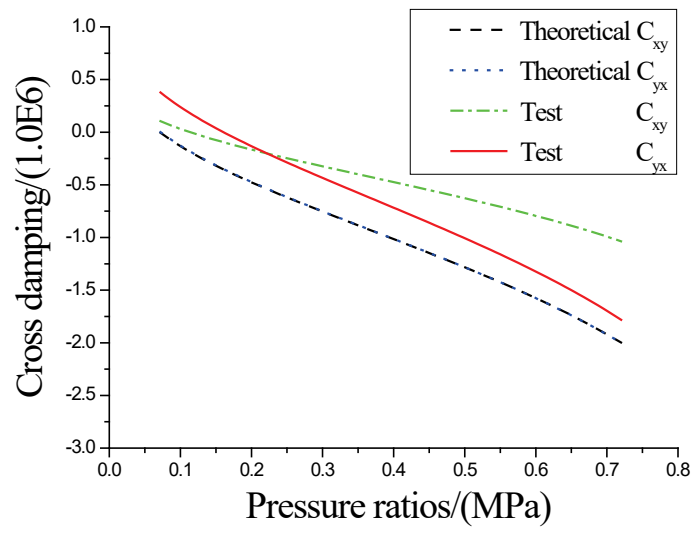

Figure 13 Cross damping coefficient under different pressure ratios

\subsection{Structural Dynamic Stiffness of HLLSJBs}

The past studies have shown that the structural dynamic stiffness of a journal bearing in the horizontal direction is less than the value in the vertical direction. Therefore, the structural dynamic stiffness in the horizontal direction is analyzed in this study. Fig. 14 shows the time and frequency domain signals of the exciting forces at the inner side in the horizontal direction that have been subjected to an excitation treatment. Fig. 15 shows the frequency spectra of the exciting forces and original response signals. It can be seen from the figures that the frequency spectrum of the exciting forces is smooth and continuous and that the signals within the main lobe are relatively credible and present a wide spectrum with good aperiodic characteristics. In addition, this frequency spectrum is the same as the spectrum of the theoretical pulse exciting force and is relatively smooth, thereby proving the reasonableness of the parameter settings and the frequency spectrum transform. It can be relatively clearly seen from the response spectrum that the structural dynamic stiffness has a first-order natural frequency of approximately $450 \mathrm{~Hz}$ and a second-order natural frequency of approximately $900 \mathrm{~Hz}$. Therefore, when a journal bearing is in operation, attention should be paid to frequencies near the resonant frequency to prevent damage and impact on the structure.
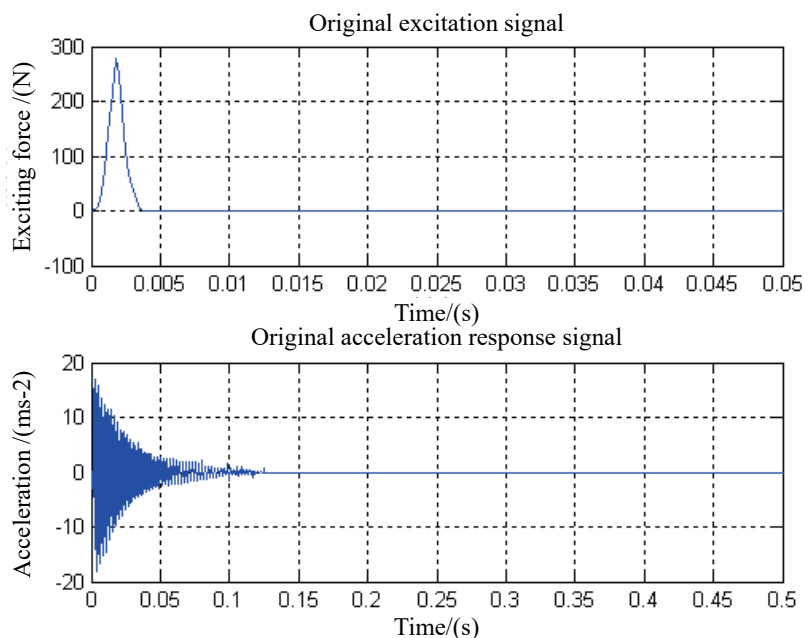

Figure 14 Time-domain chart of the excitation test at the inner side in the horizontal direction
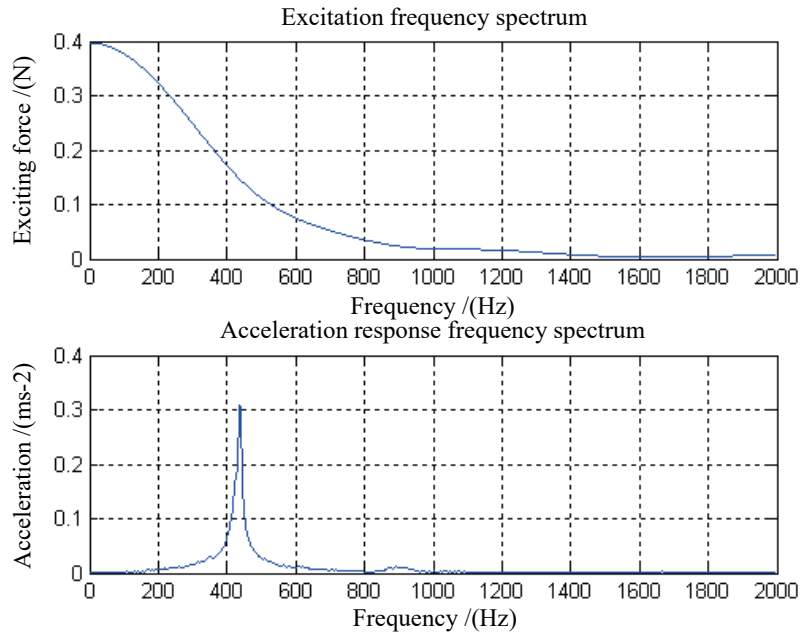

Figure 15 Frequency-domain chart of the excitation test at the inner side in the horizontal direction

The resonance peak near approximately $450 \mathrm{~Hz}$ is within the effective energy range of the exciting force. An effective bandwidth is selected near the formant based on the half-power point method to identify the stiffness of the vibration system. Each point within the selected frequency band is substituted into the least-squares equation, and thus, the horizontal structural dynamic stiffness of the journal bearing is obtained $\left(1.093 \times 10^{8} \mathrm{~N} / \mathrm{m}\right)$.

The test results show that the structural dynamic stiffness of the journal bearing in the vertical direction has an order of magnitude of $10^{8} \mathrm{~N} / \mathrm{m}$. 


\section{CONCLUSIONS}

In this study, an inverted test platform for the dynamic stiffness of the HLLSJBs is developed, by using multiple sensors, a novel dynamic calibration method and a simplified dynamic stiffness identification method with RFFT are proposed, and then the oil film dynamic stiffness and structural dynamic stiffness of the HLLSJBs are investigated in detailed.

(1) It develops an inverted test platform for the dynamic stiffness of the journal bearings of the propulsion shafting systems in ships. Based on the proposed simplified algorithm for identifying dynamic stiffness, the dynamic coefficients of journal bearings with specific structural characteristics under specific working conditions can be obtained through adjusting the relevant test conditions.

(2) It proposes a new dynamic test system calibration method. This method can directly dynamically calibrate each channel of the test system by using the floating bearing as the mass block for calibration, thereby ensuring that the calibration conditions may be consistent with the test conditions, which is beneficial for the improvement of the accuracy and precision of the test results.

(3) The test results show that under the specific test conditions (low-speed and heavy-load), the oil film dynamic stiffness of HLLSJBs has an order of magnitude of $10^{8} \mathrm{~N} / \mathrm{m}$. The test results essentially agree with the classic theoretical analysis results, thereby verifying the effectiveness and accuracy of the simplified identification method proposed in the present study.

(4) Based on the hammer impact method, the order of magnitude of the structural dynamic stiffness of the journal bearing is obtained $\left(10^{8} \mathrm{~N} / \mathrm{m}\right)$.

This study can be used to provide effective support for the vibration and coupled vibration suppression of the propulsion systems in ships.

\section{Acknowledgments}

This work was partly supported by National Natural Science Foundation of China under the grant No. 51505361, Innovative Talents Promotion Plan in Shaanxi Province (No. 2017KJXX-58), and Young Talent fund of University Association for Science and Technology in Shaanxi, China (No. 20160121).

\section{REFERENCES}

[1] Lee, D. H., Ahn, J. H., \& Koh, B. H. (2017). Fault detection of bearing systems through EEMD and optimization algorithm. Sensors, 17(11), 2477. https://doi.org/10.3390/s 17112477

[2] Antunović, R., Halep, A., Bučko, M., Perić, S., \& Vučetić, N. (2018). Vibration and Temperature Measurement Based Indicator of Journal Bearing Malfunction. Tehnički vjesnikTechnical Gazette, 25(4), 991-996. https://doi.org/10.17559/TV-20160530090714

[3] Smolnicki, T., Stańco, M., \& Pietrusiak, D. (2013). Distribution of loads in the large size bearing-problems of identification. Tehnički vjesnik-Technical Gazette, 20(5), 831-836.

[4] Tiwari, R., Lees, A., \& Friswell, M. (2004). Identification of dynamic bearing parameters: a review. The Shock and Vibration Digest, 36(2), 99-124. https://doi.org/10.1177/0583102404040173
[5] Dimond, T. W., Sheth, P. N., \& Allaire, P. E. (2009). Identification methods and test results for tilting pad and fixed geometry journal bearing dynamic coefficients-A review. Shock and vibration, 16(1), 13-43. https://doi.org/10.3233/SAV-2009-0452

[6] Babu, T. N., Raj, T. M., \& Lakshmanan, T. (2014). Application of Butterworth filter for fault diagnosis on journal bearing. Journal of Vibroengineering, 16(3), 16021617.

[7] Carlton, J. (2012). In Marine Propellers and Propulsion. Butterworth-Heinemann: Oxford, England. https://doi.org/10.1016/B978-0-08-097123-0.00002-2

[8] Komar, I., Vulić, N., \& Roldo, L. (2013). Hydrodynamic and elastohydrodynamic lubrication model to verify the performance of marine propulsion shafting. Transactions of Famena, 37(1), 15-27.

[9] Tiwari, R. \& Chakravarthy, V. (2006). Simultaneous identification of residual unbalances and bearing dynamic parameters from impulse responses of rotor-bearing systems. Mechanical Systems and Signal Processing, 20(7), 15901614. https://doi.org/10.1016/j.ymssp.2006.01.005

[10] Żywica, G. (2016). An experimental investigation conducted in order to determine bearing dynamic coefficients of two hydrodynamic bearings using impulse responses. Transactions of the Institute of Fluid-Flow Machinery, 133, 39-54.

[11] Schwartz, B., Fittro, R., \& Knospe, C. (2017, June). Understanding the Effect of Systematic Errors on the Accuracy of Experimental Measurements of Fluid-Film Bearing Dynamic Coefficients. In ASME Turbo Expo 2017: Turbomachinery Technical Conference and Exposition (pp. V07AT34A028-V07AT34A028). American Society of Mechanical Engineers.

[12] Li, Q., Zhang, S., \% Ma, L. (2017). Stiffness and damping coefficients for journal bearing using the 3D transient flow calculation. Journal of Mechanical Science and Technology, 31(5), 2083-2091. https://doi.org/10.1007/s12206-017-0405-9

[13] Song, Q., Sun, F., \& Ma, J. (2002). A New Algorithm and Experimental Investigation for the Identification of Dynamic Characteristics of Journal Bearings. Journal of Beijing Institute of Technology, 22(6), 682-686.

[14] Yuan, X., Wang, H., \& Gao, Y. (1992). A Study on the Oil Film Stiffness and Damping Coefficients of Hydrodynamic Journal Bearings. Journal of Mechanical Engineering, 28(5), 83-87.

[15] Lin, J.,Qiu, D., \& Liu, B. (1981).Research into the Procedure of Experimentally Determining the Oil-Film Stiffness and Damping Characteristics in Hydrodynamic Journal Bearings. Journal of Xi'an Jiaotong University, 15(2), 127143.

[16] Garg, H., Sharda, H., \& Kumar, V. (2006). On the design and development of hybrid journal bearings: a review. Tribotest, 12(1), 1-19. https://doi.org/10.1002/tt.1

Contact information

1) Junning LI, Ass. Prof., Mechanical Engineering (Corresponding author) 2) Ka HAN, Mechanical Engineering

School of Mechatronic Engineering, Xi'an Technological University,

No. 2, Xuefu Road, Xi'an, Shannxi, 710021, P. R. China

1)E-mail: junningli@outlook.com

2) E-mail: xatuhk@126.com

Wei CHEN, Prof., Mechanical Engineering

Key Laboratory of Education Ministry for Modern Design and Rotor-Bearing

System, School of Mechanical Engineering, Xi'an Jiaotong University,

No. 28, Xianning West Road, Xi'an, Shannxi, 710049, P. R. China

E-mail: xjtuchenw@hotmail.com 